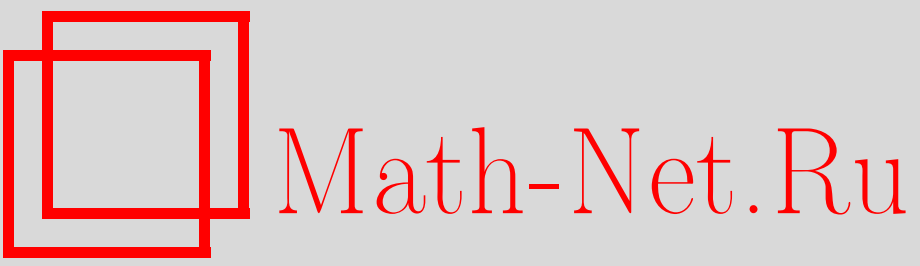

П. Г. Эстевес, Ч. Чу, Разделение переменных в нелинейных волновых уравнениях с переменной волновой скоростью, ТМФ, 2002, том 133, номер 2, 202-210

DOI: https://doi.org/10.4213/tmf390

Использование Общероссийского математического портала Math-Net.Ru подразумевает, что вы прочитали и согласны с пользовательским соглашением

http://www . mathnet.ru/rus/agreement

Параметры загрузки:

IP: 54.224 .187 .69

26 апреля 2023 г., 13:42:54 
ТЕОРЕТИЧЕСКАЯ

И МАТЕМАТИЧЕСКАЯ

ФИЗИКА

Том 133, № 2

ноябрь, 2002

(C) 2002 r.

П.Г. Эстевес ${ }^{*}$ Ч.-Ж. Чу ${ }^{\dagger}$

\section{РАЗДЕЛЕНИЕ ПЕРЕМЕННЫХ В НЕЛИНЕЙНЫХ ВОЛНОВЫХ УРАВНЕНИЯХ С ПЕРЕМЕННОЙ ВОЛНОВОЙ СКОРОСТЬЮ}

Развит обобщенный условно-симметрийный подход к функциональному разделению переменных в нелинейном волновом уравнении с нелинейной волновой скоростью. Этот подход позволяет получить ряд новых нелинейных волновых уравнений в размерности $1+1$ с переменными волновыми скоростями, допускающих функционально разделимые решения. Найдены точные решения полученных уравнений.

Ключевые слова: симметрии Ли, обобщенные симметрии, уравнение диффузии, нелинейные уравнения.

Классическая теория Ли симметрий дифференциальных уравнений [1], [2] является мошным методом разделения переменных в линейных дифференциальных уравнениях с частными производными [3]. Недавно по крайней мере два интересных расширения теории Ли - неклассический метод [4] и обобшенный условно-симметрийный подход [5]-[7] - были использованы при изучении функционального разделения переменных в нелинейных уравнениях диффузии с членами, учитывающими конвекцию и источник [8], [9]. Другим интересным примером является нелинейное волновое уравнение с переменной волновой скоростью

$$
u_{t t}=\left(B(u) u_{x}\right)_{x}+A(u), \quad B(u) \neq \text { const }
$$

которое имеет важные применения в теории распространения волн и в прикладных науках.

В настояшей работе мы применяем обобшенный условно-симметрийньй метод для изучения функционального разделения переменных в уравнении (1). Решение уравнения (1) называется функционально разделимым, если сушествуют функции $q(u), \phi(t)$ и $\psi(x)$ такие, что

$$
q(u)=\phi(t)+\psi(x)
$$

Классическое аддитивно разделимое решение и разделимое решение в виде произведения являются частными случаями указанного функционально разделимого решения.

* Area de Fisica Teórica, Facultad de Ciencias, Universidad de Salamanca, Salamanca, Spain

† Department of Mathematics, Northwest University, Xi'an, P. R. China 
Для случая $B=1$ функциональное разделение переменных в уравнении (1) обсуждалось несколькими авторами с использованием различных методов [10]. Уравнения, допускающие разделимые решения, включают уравнения Буллоу-Додда, уравнения sinГордон и sh-Гордон. Случай $A=0$, когда $B=e^{a u}$ или $B=u^{a}$, обсуждался в работе [11] с использованием классического метода Ли.

ОПРЕДЕЛЕНИЕ. Эволюционное векторное поле

$$
V=\eta(t, x, u, \ldots) \frac{\partial}{\partial u}
$$

является обобшенной условной симметрией уравнения (1), если

$$
\left.V^{(2)}\left(u_{t t}-\left(B(u) u_{x}\right)_{x}-A(u)\right)\right|_{E \cap W}=0,
$$

где $E$ - многообразие решений уравнения (1), $W$ - система второго порядка для (1), полученная добавлением условия инвариантной поверхности $\eta=0$ и его частных производных по $x$, a $V^{(2)}$ является вторым продолжением инфинитезимального оператора $V$.

Важный факт состоит в том, что если уравнение (1) допускает обобщенную условную симметрию

$$
V=\left(u_{x t}+G(u) u_{x} u_{t}\right) \frac{\partial}{\partial u}, \quad G=\frac{q^{\prime \prime}(u)}{q^{\prime}(u)},
$$

то оно имеет функционально разделимое решение (2).

Непосредственное вычисление дает

$$
\begin{aligned}
V^{(2)}\left(u_{t t}-\right. & \left.\left(B(u) u_{x}\right)_{x}-A(u)\right)\left.\right|_{E \cap W}= \\
= & 3\left(B^{\prime \prime}-G B^{\prime}-\frac{B^{\prime 2}}{B}\right) u_{t} u_{x} u_{x x}+\left[G^{\prime \prime}-2 G G^{\prime}-\frac{B^{\prime}}{B}\left(G^{\prime}-G^{2}\right)\right] u_{x} u_{t}^{3}+ \\
& +\left[B^{\prime \prime \prime}-\frac{B^{\prime}}{B}\left(B^{\prime \prime}+G B^{\prime}\right)-B\left(G^{\prime \prime}-2 G G^{\prime}\right)-G B^{\prime \prime}-G^{2} B^{\prime}\right] u_{t} u_{x}^{3}+ \\
& +\left[A^{\prime \prime}+\left(G-\frac{B^{\prime}}{B}\right) A^{\prime}+\left(3 G^{\prime}-2 G^{2}-\frac{B^{\prime}}{B} G\right) A\right] u_{t} u_{x} .
\end{aligned}
$$

Условие обращения в нуль этого выражения дает уравнения на $B, G$ и $A$ :

$$
\begin{gathered}
B^{\prime \prime}-G B^{\prime}-\frac{B^{\prime 2}}{B}=0, \\
G^{\prime \prime}-2 G G^{\prime}-\frac{B^{\prime}}{B}\left(G^{\prime}-G^{2}\right)=0 \\
A^{\prime \prime}+\left(G-\frac{B^{\prime}}{B}\right) A^{\prime}+\left(3 G^{\prime}-2 G^{2}-\frac{B^{\prime}}{B} G\right) A=0 .
\end{gathered}
$$

Для уравнения (5б) рассмотрим два случая. 
Случай 1. $G^{\prime}-G^{2}=0$. В этом случае $G$ имеет вид

$$
G=0 \text { или } G=-\frac{1}{u+u_{0}},
$$

где трансляциями $u$ можно выбрать $u_{0}$ равным нулю. При $G=0$ мы получаем классическое аддитивно разделимое решение, а при $G=-1 / u$ - разделимое решение в виде произведения. Рассмотрим эти случаи по отдельности.

Случай 1a. $G=-1 / u$. Подставляя $G=-1 / u$ в (5a) и (5в), находим после скейлинга для $u$, что

$$
B=u^{a}, \quad a=\text { const }
$$

а $A$ удовлетворяет уравнению

$$
A^{\prime \prime}-\frac{a+1}{u} A^{\prime}+\frac{a+1}{u^{2}} A=0,
$$

решение которого есть

$$
A=c_{1} u+c_{2} u^{a+1} .
$$

Здесь и далее $c_{1}$ и $c_{2}-$ произвольные константы. Более того, из (4) следует, что $q=\ln u$. Таким образом, мы установили, что уравнение

$$
u_{t t}=\left(u^{a} u_{x}\right)_{x}+c_{1} u+c_{2} u^{a+1}
$$

допускает разделимое решение в виде произведения

$$
u=\phi(t) \psi(x),
$$

где $\phi(t)$ и $\psi(x)$ удовлетворяют системе

$$
\begin{gathered}
\phi^{\prime \prime}-c_{1} \phi-\lambda \phi^{a+1}=0, \\
\left(\psi^{a} \psi^{\prime}\right)^{\prime}+c_{2} \psi^{a+1}-\lambda \psi=0,
\end{gathered}
$$

$\lambda$ обозначает постоянную разделения. Уравнение (8а) можно проинтегрировать как

$$
\begin{aligned}
\phi^{2}-c_{1} \phi^{2}-\frac{2 \lambda}{a+2} \phi^{a+2} & =d_{1}, \text { если } a \neq-2, \\
\phi^{\prime 2}-c_{1} \phi^{2}-2 \lambda \ln \phi & =d_{1}, \text { если } a=-2 .
\end{aligned}
$$

Первый интеграл уравнения (8б) имеет вид

$$
\begin{aligned}
& \psi^{\prime 2}+\frac{c_{2}}{a+1} \psi^{2}-\frac{2 \lambda}{a+2} \psi^{2-a}=d_{2} \psi^{-2 a} \text {, если } a \neq-2, \quad a \neq-1, \\
& \psi^{\prime 2}-c_{2} \psi^{2}-2 \lambda \psi^{4} \ln \psi=d_{2} \psi^{4}, \quad \text { если } a=-2 \text {, } \\
& \psi^{\prime 2}+2 c_{2} \psi^{2} \ln \psi-2 \lambda \psi^{3}=d_{2} \psi^{2}, \quad \text { если } a=-1 \text {. }
\end{aligned}
$$

Соответственно решения для $\phi$ и $\psi$ можно неявно записать в следуюшем виде. 
1а.1. Если $a \neq-2,-1$,

$$
\begin{gathered}
\int^{\phi(t)} \frac{d z}{\sqrt{2 \lambda z^{2+a} /(a+2)+c_{1} z^{2}+d_{1}}}=t, \\
\int^{\psi(x)} \frac{d y}{\sqrt{2 \lambda y^{2-a} /(a+2)-c_{2} y^{2} / a+1+d_{2} y^{-2 a}}}=x .
\end{gathered}
$$

1a.2. Если $a=-2$,

$$
\begin{aligned}
\int^{\phi(t)} \frac{d z}{\sqrt{c_{1} z^{2}+2 \lambda \ln z+d_{1}}} & =t, \\
\int^{\psi(x)} \frac{d y}{\sqrt{2 \lambda y^{4} \ln y+c_{2} y^{2}+d_{2} y^{4}}} & =x .
\end{aligned}
$$

1a.3. Если $a=-1$,

$$
\begin{aligned}
\int^{\phi(t)} \frac{d z}{\sqrt{c_{1} z^{2}+2 \lambda z+d_{1}}} & =t, \\
& =x . \\
\sqrt{2 \lambda y^{3}-2 c_{2} y^{2} \ln y+d_{2} y^{2}} & =x .
\end{aligned}
$$

Случай 1б. $G=0$. При этом имеем

$$
B=e^{u}, \quad A=c_{1}+c_{2} e^{u}, \quad q=u .
$$

Получаем, что уравнение

$$
u_{t t}=\left(e^{u} u_{x}\right)_{x}+c_{1}+c_{2} e^{u}
$$

допускает аддитивно разделимое решение

$$
u=\phi(t)+\psi(x)
$$

где $\phi(t)$ и $\psi(x)$ удовлетворяют системе

$$
\begin{gathered}
\phi^{\prime \prime}-c_{1}-\lambda e^{\phi}=0, \\
\left(e^{\psi} \psi^{\prime}\right)^{\prime}+c_{2} e^{\psi}-\lambda=0,
\end{gathered}
$$

которую можно проинтегрировать следующим образом.

1б.1. Если $c_{2}>0$,

$$
\begin{aligned}
& \int^{\phi(t)} \frac{d z}{\sqrt{2 \lambda e^{z}+2 c_{1} z+d_{1}}}=t, \\
& \psi(x)=\ln \left[\frac{\lambda}{c_{2}}-d_{2} \cos \sqrt{c_{2}} x\right] .
\end{aligned}
$$


1б.2. Если $c_{2}<0$,

$$
\begin{gathered}
\int^{\phi(t)} \frac{d z}{\sqrt{2 \lambda e^{z}+2 c_{1} z+d_{1}}}=t, \\
\psi(x)=\ln \left[\frac{\lambda}{c_{2}}-d_{2} \operatorname{ch} \sqrt{-c_{2}} x\right] .
\end{gathered}
$$

Случай 2. $G^{\prime}-G^{2} \neq 0$. Можно определить $h(u)$ так, чтобы

$$
G=-\frac{h^{\prime}}{h} \Longrightarrow q^{\prime} \sim \frac{1}{h}
$$

Из уравнения (5б) находим

$$
B=\frac{B_{0} h^{\prime \prime}}{h},
$$

где $B_{0}$ - постоянная, которую можно выбрать равной \pm 1 с помошью скейлинга переменной $t$. Подставляя $G=-h^{\prime} / h$ в (5a), мы находим, что $B^{\prime} / B=a / h, a=$ const, так что $B$ можно выразить в терминах $h$ с помошью соотношения

$$
\frac{B^{\prime}}{B}=\frac{a}{h}
$$

Очевидно, $h$ удовлетворяет уравнению

$$
h h^{\prime \prime \prime}-h^{\prime} h^{\prime \prime}=a h^{\prime \prime}
$$

которое можно проинтегрировать как

$$
h h^{\prime \prime}-h^{\prime 2}=a h^{\prime}+b .
$$

Отметим, что в дальнейшем мы явно предполагаем, что $a \neq 0$, поскольку, как легко видеть из уравнения (13), предположение $a=0$ влечет за собой $B=$ const, что соответствует случаю, широко изучавшемуся в работах [10], [11].

При $a \neq 0$ имеются два случая.

Случай 2а. $b=0$. Решая уравнение (14), мы приходим к соотношению

$$
h=\frac{a}{d}+c e^{d u}
$$

где $d$ - произвольная постоянная. Отсюда получаем

$$
B=-\frac{B_{0} d^{2} e^{d u}}{a /(d c)+e^{d u}}
$$

С помощью скейлинга и трансляции для $u$ можно положить $d= \pm 1$ и $a / c= \pm 1$. Выделяются четыре возможности. 
2a.1. $B_{0}=-d=1, a / c=1$. В этом случае

$$
B=\frac{1}{e^{u}-1}, \quad h=c\left(e^{-u}-1\right), \quad q \sim \ln \left(e^{u}-1\right)
$$

После подстановки $B$ и $h$ в уравнение (5в) видно, что $A$ удовлетворяет уравнению

$$
A^{\prime \prime}+A^{\prime}+\frac{2}{e^{u}-1} A=0
$$

которое имеет общее решение

$$
A(u)=c_{1}\left[1-2 e^{-u}+2\left(e^{-u}-e^{-2 u}\right) \ln \left(e^{u}-1\right)\right]+c_{2}\left(e^{-2 u}-e^{-u}\right) .
$$

Таким образом, мы установили, что уравнение

$$
u_{t t}=\left(\frac{u_{x}}{e^{u}-1}\right)_{x}+c_{1}\left[1-2 e^{-u}+2\left(e^{-u}-e^{-2 u}\right) \ln \left(e^{u}-1\right)\right]+c_{2}\left(e^{-2 u}-e^{-u}\right)
$$

допускает функционально разделимое решение

$$
u=\ln [1+\phi(t) \psi(x)]
$$

Подстановка (16) в (15) показьвает, что функции $\phi(t)$ и $\psi(x)$ удовлетворяют системе

$$
\begin{aligned}
\phi^{\prime 2} & =2 c_{1} \phi^{2} \ln \phi+\left(\alpha-c_{1}\right) \phi^{2}-2 \lambda \phi-\beta, \\
\psi^{\prime 2} & =2 c_{1} \psi^{2} \ln \psi+\beta \psi^{4}-2 \lambda \psi^{3}+\left(c_{1}-c_{2}-\alpha\right) \psi^{2}
\end{aligned}
$$

где $\lambda, \alpha$ и $\beta$ - произвольные постоянные.

Неявное решение системы (17) имеет вид

$$
\begin{aligned}
\int^{\phi(t)} \frac{d z}{\sqrt{2 c_{1} z^{2} \ln z+\left(\alpha-c_{1}\right) z^{2}-2 \lambda z-\beta}} & =t, \\
\frac{d y}{\sqrt{2 c_{1} y^{2} \ln y+\beta y^{4}-2 \lambda y^{3}+\left(c_{1}-c_{2}-\alpha\right) y^{2}}} & =x .
\end{aligned}
$$

Оставшиеся случаи исследуются аналогично. Запишем результаты следующим образом. 
2a.2. $B_{0}=-d=-1, a / c=1$. В этом случае

$$
B=\frac{e^{u}}{1+e^{u}}, \quad h=c\left(1+e^{u}\right), \quad q=u-\ln \left(1+e^{u}\right) .
$$

Получающееся уравнение имеет вид

$$
u_{t t}=\left(\frac{e^{u}}{e^{u}+1} u_{x}\right)_{x}-c_{1}\left[1+2 e^{u}+2\left(e^{u}+e^{2 u}\right) \ln \frac{e^{u}}{e^{u}+1}\right]+c_{2}\left(e^{2 u}+e^{u}\right),
$$

а функционально разделимое решение есть

$$
u=\ln \left[\frac{1}{\phi(t) \psi(x)-1}\right] .
$$

Обыкновенные дифференциальные уравнения для $\phi$ и $\psi$ имеют вид

$$
\begin{aligned}
\phi^{\prime 2} & =2 c_{1} \phi^{2} \ln \phi+\left(\alpha-c_{1}\right) \phi^{2}-2 \lambda \phi-\beta, \\
\psi^{\prime 2} & =-2 c_{1} \psi^{2} \ln \psi-\beta \psi^{4}-2 \lambda \psi^{3}+\left(\alpha-c_{1}-c_{2}\right) \psi^{2}
\end{aligned}
$$

и могут быть проинтегрированы как

$$
\int^{\psi(x)} \frac{d z}{\sqrt{-2 c_{1} y^{2} \ln y-\beta y^{4}-2 \lambda y^{3}+\left(\alpha-c_{1}-c_{2}\right) y^{2}}}=x .
$$

2а.3. $B_{0}=-d=-1, a / c=-1$. В этом случае

$$
\begin{gathered}
B=\frac{e^{u}}{e^{u}-1}, \quad h=c\left(e^{u}-1\right), \quad q=-u+\ln \left(e^{u}-1\right), \\
u_{t t}=\left(\frac{e^{u}}{e^{u}-1} u_{x}\right)_{x}+c_{1}\left[2 e^{u}-1-2\left(e^{2 u}-e^{u}\right) \ln \frac{e^{u}}{e^{u}-1}\right]+c_{2}\left(e^{2 u}-e^{u}\right), \\
u=\ln \left[\frac{1}{1-\phi(t) \psi(x)}\right] .
\end{gathered}
$$

Уравнениями для $\phi$ и $\psi$ являются также уравнения (21). Поэтому решения для $\phi$ и $\psi$ даются формулами (22).

2a.4. $B_{0}=d=-1, a / c=-1$. В этом случае

$$
\begin{gathered}
B=\frac{1}{1+e^{u}}, \quad h=c\left(1+e^{-u}\right), \quad q=\ln \left(1+e^{u}\right), \\
u_{t t}=\left(\frac{u_{x}}{e^{u}+1}\right)_{x}+c_{1}\left[2 e^{-u}+1-2\left(e^{-2 u}+e^{-u}\right) \ln \left(e^{u}+1\right)\right]-c_{2}\left(e^{-2 u}+e^{-u}\right), \\
u=\ln [\phi(t) \psi(x)-1] .
\end{gathered}
$$

Как и в предыдушем случае, $\phi$ и $\psi$ должны удовлетворять уравнениям (21), а его решения явно даются формулами (22). 
Случай $\mathbf{2 b .} b \neq 0$. Полагая $H(h)=h_{u}$ в $(6)$, получаем, что $H$ удовлетворяет уравнению

$$
h H H_{h}=H^{2}+a H+b,
$$

которое интегрируется как

$$
\int^{H} \frac{z d z}{z^{2}+a z+b}=\ln \left(\frac{h}{h_{0}}\right), \quad h_{0}=\text { const. }
$$

Далее для $\Delta \equiv a^{2}-4 b$ следует рассмотреть три случая по отдельности.

2б.1. $\Delta=0$. Уравнение (27) интегрируется как

$$
\left(h^{\prime}+\frac{a}{2}\right) e^{a /\left(2 h^{\prime}+a\right)}=\frac{h}{h_{0}},
$$

так что $h$ удовлетворяет уравнению

$$
H \equiv h^{\prime}=f_{1}(h)
$$

2б.2. $\Delta>0 \Longrightarrow \Delta=c^{2}$. В этом случае уравнение (27) интегрируется как

$$
\left(h^{\prime}+\frac{a+c}{2}\right)^{c+a}\left(h^{\prime}+\frac{a-c}{2}\right)^{c-a}=\left(\frac{h}{h_{0}}\right)^{2 c} .
$$

Перепишем его в неявном виде:

$$
h^{\prime}=f_{2}(h) \text {. }
$$

2б.3. $\Delta<0 \Longrightarrow \Delta=-c^{2}$. В этом случае (27) интегрируется как

$$
\left[1+\left(\frac{2 h^{\prime}+a}{c}\right)^{2}\right] \exp \left[-2 \frac{a}{c} \operatorname{arctg}\left(\frac{2 h^{\prime}+a}{c}\right)\right]=\left(\frac{h}{h_{0}}\right)^{2} .
$$

Перепишем его в неявном виде как

$$
h^{\prime}=f_{3}(h)
$$

В каждом случае $h$ можно неявно определить посредством формулы

$$
\int^{h} \frac{d z}{f_{i}(z)}=u
$$

a $B$ имеет вид

$$
B_{i}(u)=-\frac{f_{i}^{2}+a f_{i}+b}{h^{2}}, \quad i=1,2,3
$$

и для $A$ выполнено

$$
h^{2} A^{\prime \prime}-h\left(f_{i}+a\right) A^{\prime}-\left(2 f_{i}^{2}+2 a f_{i}+3 b\right) A=0 .
$$

Соответствуюшее уравнение имеет разделимое решение вида

$$
\int^{u} \frac{d z}{h(z)}=\phi(t)+\psi(x)
$$

3 Теоретическая и математическая физика, т. 133, № 2, 2002 г. 


\section{ЗАКЛЮЧЕНИЕ}

Мы применили обобшенный условно-симметрийный подход для получения функционально разделимых решений. Следует отметить, что эти разделимые решения нельзя получить с использованием классического симметрийного метода Ли. Излагаемый подход можно развивать далее для изучения высших нелинейных волновых уравнений.

Легко видеть, что явное поведение решений зависит от выбора многих произвольных постоянных. Это означает, что имеется множество различных случаев. Явное исследование этих случаев и их физического и математического значения выходит за рамки данной работы и является предметом дальнейших исследований.

Благодарности. Данная работа частично поддержана со стороны DGICYT (проект РВ98-0262). Ч.Ж. Чу благодарит Министерство образования и культуры Испании за предоставленную стипендию.

\section{Список литературы}

[1] G. W. Bluman, S. Kumei. Symmetries and Differential Equations. New York: Springer, 1989.

[2] П. Олвер. Приложения групп Ли к дифференциальным уравнениям. М.: Мир, 1989.

[3] W. Miller. Symmetry and Separation of Variables. Reading, MA: Addison-Wesley, 1977.

[4] G. W. Bluman, J. D. Cole. J. Math. Mech. 1969. V. 18. P. 1025.

[5] A. S. Fokas, Q. M. Liu. Phys. Rev. Lett. 1994. V. 72. P. 3293.

[6] R. Z. Zhdanov. J. Phys. A. 1995. V. 28. P. 3841.

[7] C. Z. Qu. Stud. Appl. Math. 1997. V. 99. P. 107.

[8] E. Pucci, G. Saccomandi. Physica D. 2000. V. 139. P. 28.

[9] C. Z. Qu, S. L. Zhang, R. C. Liu. Physica D. 2000. V. 144. P. 97.

[10] W. Miller, L. A. Rubel. J. Phys. A. 1993. V. 26. P. 1901; A. M. Grundland, E. Infeld. J. Math. Phys. 1992. V. 33. P. 2498; R. Z. Zhdanov. J. Phys. A. 1994. V. 27. P. L291.

[11] W. F. Ames, R. J. Lohner, E. Adams. Int. J. Nonlinear Mech. 1981. V. 16. P. 439. 